\title{
Processus de transfert superficiel des produits phytosanitaires, de la parcelle au bassin versant
}

\author{
Superficial transfer process of pesticides, from the plot of land \\ to the catchment area
}

par J.J. Gril, V. Gouy, N. Carluer

Cemagref, Lyon

Runoff and diverse other forms of low depth flow generate transfers towards catchment areas of subtances which are partly due to agriculture practices : nitrogen, phosphorus, pesticides, suspended matter. They are at the origin of pollution in surface water. Numerous factors determine the future and the transfer attenuation from the source to the rivers of these products. The quantification of the processes is still a problem and is a theme of research.

\section{I $\square$ INTRODUCTION}

L'eau de pluie interceptée par un impluvium se partage suivant des chemins divers que l'on peut ramener pour simplifier à trois catégories : le ruissellement, les écoulements subsuperficiels (à faible profondeur) et l'infiltration vers les couches plus profondes. L'importance relative de ces voies dépend à la fois des caractéristiques du climat et de celles du bassin versant qui intercepte la pluie.

En suivant ces différents chemins, l'eau entraine des produits variés, susceptibles de créer des perturbations dans le milieu aquatique et d'être dommageables pour les usages de l'eau. Dans le cas des territoires agricoles, ce sont principalement les matières en suspension produites par l'érosion, l'azote, le phosphore et les produits phytosanitaires. Nous traiterons ici de ces derniers.

La diversité des chemins suivis par l'eau et la complexité des interactions entre ces substances et le milieu font que de nombreux facteurs déterminent leur devenir et l'atténuation de leur transfert depuis leur source d'émission jusqu'aux rivières et plans d'eau.

Dans le cadre de cette communication, nous nous intéresserons plus spécialement aux transferts qui suivent les voies superficielles : ruissellement et écoulements à faible profondeur, en laissant de côté l'infiltration vers les couches profondes. Nous traiterons cette question en nous plaçant successivement à l'échelle de la parcelle agricole, du versant et du bassin versant. Nous nous efforcerons également d'identifier les principales zones d'ombre qui restent à éclaircir pour que les connaissances débouchent sur des outils opérationnels de maîtrise de ces pollutions.

\section{II $\square$ LA PARCELLE AGRICOLE}

\subsection{Le cheminement de l'eau}

Le ruissellement de surface dit " hortonien " se produit quand l'intensité de la pluie dépasse la capacité d'infiltration de la surface du sol. L'occurrence et l'importance de ce ruissellement dépendent de très nombreux facteurs, en rapport avec les caractéristiques de la précipitation et de celles du sol et de sa couverture. L'eau qui s'infiltre sous la surface peut continuer son trajet vers les couches profondes ou être, au moins partiellement, arrêtée par un horizon imperméable à faible profondeur et s'écouler à la surface de ce dernier (dans le premier mètre, pour simplifier) : on parle alors d'écoulement subsuperficiel ou hypodermique. Si l'eau s'accumule au point de saturer l'horizon de surface et de déborder apparaît alors le ruissellement par saturation (figure 1).

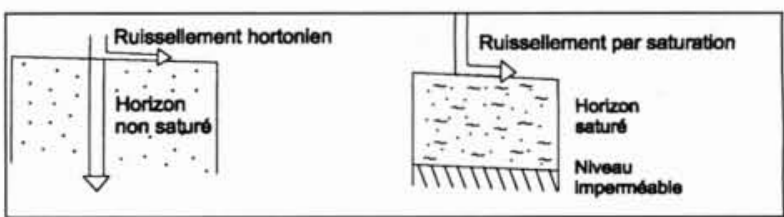

1. Ruissellements hortonien et par saturation.

Le ruissellement qui se forme à la surface du sol s'organise et se concentre progressivement en suivant les dépressions.

L'eau qui s'infiltre sous la surface suit deux types de chemin : une circulation lente (capillaire) dans la microporosité du sol, et une circulation rapide dans les macropores et fissures dont la dimension autorise les mouvements gravitaires. 
Cela se produit aussi bien en conditions naturelles qu'au sein d'une parcelle cultivée. Mais la mise en culture complique considérablement ces phénomènes en introduisant de nombreuses perturbations dont nous citerons les principales.

— Dégradation de la perméabilité de surface, sous l'effet :

- de l'absence ou de la faible importance de la couverture végétale pendant une période plus ou moins importante de l'année,

- de la fragilisation de la surface du sol sous l'effet d'une chute fréquente du taux de matière organique (instabilité structurale), de la finesse de préparation du lit de semence, ...

- de la compaction par les engins agricoles (traces de roues) ;

- Phénomène inverse d'augmentation (brutale) de la perméabilité de surface lors de certains travaux (labour, déchaumage, ...) ;

- Tassement à faible profondeur, dû aux travaux agricoles (semelle de labour), favorisant les écoulements subsuperficiels ;

- Modification des écoulements de surface, sous l'effet du microrelief cultural (traces de roues, lignes de semis, dérayures) dont l'influence est d'autant plus sensible que la pente est plus faible.

- Rôle du drainage par drains enterrés, qui produit une forme particulière d'écoulement subsuperficiel rapide et réduit la part du ruissellement.

Globalement et en général, la mise en culture augmente l'importance du ruissellement, dont le volume annuel reste néanmoins habituellement inférieur à celui de l'infiltration. Le ruissellement de surface strict n'atteint des proportions relativement importantes que dans des périodes courtes, et l'extension en France du drainage a probablement réduit assez significativement le ruissellement dans les secteurs concernés.

\subsection{Le transfert des substances}

Le ruissellement produit un arrachement des particules de terre, tout d'abord diffus et généralement peu visible : cette érosion, dite elle-même diffuse, concerne essentiellement les particules fines et la matière organique.

Puis, à la faveur de la concentration progressive des filets d'eau, apparaissent les griffes et les ravines qui creusent plus ou moins profondément les couches superficielles du sol en emportant des matériaux de granulométrie plus variée. Conjointement, il apparaît souvent des dépôts au sein des parcelles, provoqués par le ralentissement et l'infiltration de l'eau dans des zones concaves ; cela provoque le dépôt des particules grossières, et la terre érodée qui est évacuée hors de la parcelle est plus riche en fines et en matière organique que le sol superficiel en place.

Le vecteur principal du transfert du phosphore est le ruissellement, la dynamique de cet élément étant surtout liée à celle des particules fines. Le transfert de l'azote est, à l'inverse, plutôt lié à l'infiltration.

Le cas des produits phytosanitaires est plus complexe : ces molécules sont très nombreuses (environ 900 homologuées en France, dont 200 d'usage courant) et leur capacité d'adsorption sur le sol et les matières en suspension est très variable. Par ailleurs, leur persistance, également très variable, joue également un rôle essentiel dans leur devenir.

\subsubsection{La mobilité des produits phytosanitaires}

A priori, la mobilité des produits phytosanitaires est en rapport avec deux caractéristiques : la solubilité et le coefficient de partage eau-sol $K d$ (ou $K o c=K d /$ carbone organique). En pratique, seul le coefficient de partage se révèle être un bon indicateur de la mobilité de ces molécules. L'étude des transferts met bien en lumière une cohérence entre les concentrations observées dans le milieu aquatique et la solubilité ; toutefois, les concentrations de produits rencontrées dans les eaux naturelles sont généralement bien inférieures à leur solubilité. Même les substances peu solubles sont donc susceptibles d'être entraînées par l'eau à des concentrations faibles, mais significatives (en particulier au regard des contraintes réglementaires).

Par ailleurs les produits sont assez rarement appliqués dans le sol par enfouissement, mais le plus souvent sur les plantes et/ou sur la surface du sol.

De plus, comme cela a été signalé ci-dessus, l'érosion diffuse est habituellement peu importante en terme de quantité et, ainsi, assez peu de matières en suspension sont disponibles pour fixer les substances immédiatement après leur " prise en charge " par l'eau de pluie interceptée par le sol.

Il résulte de tout ceci que la fraction dissoute représente le plus souvent l'essentiel de l'entraînement de départ. Si l'érosion linéaire se manifeste plus en aval, le ruissellement s'enrichit alors en matières en suspension disponibles pour fixer les molécules et déplacer l'équilibre dans le sens d'une plus grande adsorption particulaire.

Ainsi, être caractérisée par un $K d$ élevé n'empêche pas une molécule d'être entraînée par le ruissellement. Les colloïdes organiques présents dans l'eau contribuent également à favoriser ce phénomène, du fait de l'affinité des produits pour ces substances. Toutefois, en permettant une fixation plus importante sur le sol en place, une forte capacité d'adsorption limite cet entraînement, à condition que la rétention sur le sol soit effectivement possible : par exemple, le produit intercepté par une trace de roue lisse, offrant une faible surface de contact et une perméabilité réduite, risque d'être facilement mobilisé dans le ruissellement quel que soit son $K d$.

Ce dernier joue en revanche un rôle déterminant vis-à-vis de l'entraînement des molécules par l'infiltration : l'importance de la surface de contact offerte par le substrat va permettre la fixation et, cette fois, opérer une discrimination très significative entre molécules à faible et fort $K d$. Cela n'est toutefois rigoureusement vrai que pour la circulation de

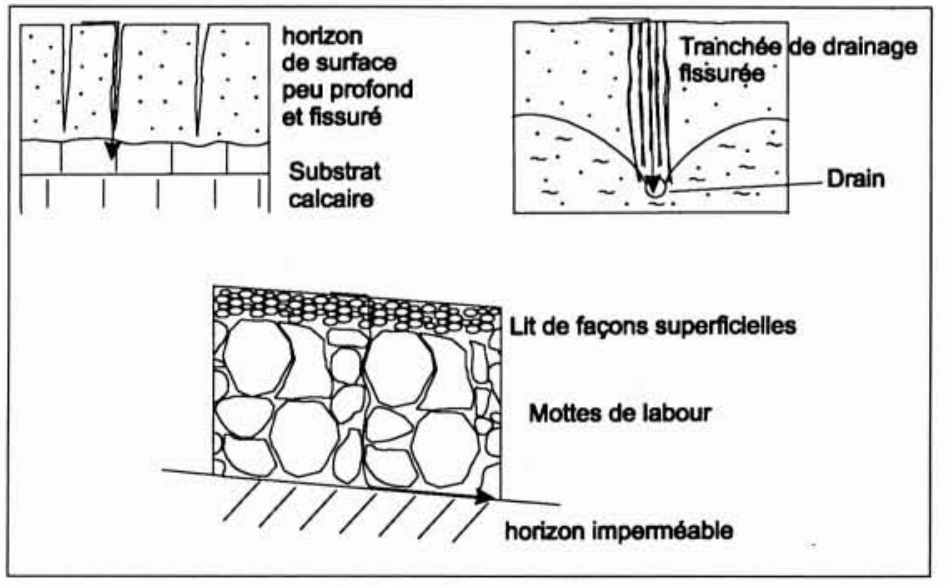

2. Transferts rapides. 
l'eau dans la microporosité et, probablement pour la faible macroporosité $(<0,5 \mathrm{~mm})$ [1]. L'eau qui circule rapidement dans des fissures de dimension plus importante entraînera les produits en profondeur même si leur $K d$ est élevé (figure 2).

Ainsi, on peut identifier au moins trois cas où le transfert sous la surface du sol peut être conséquent pour des molécules à $K d$ élevé :

— sous un sol à forte macroporosité et peu profond sur un substrat rocheux faillé,

- en présence d'un réseau de drains et si le sol est resté fissuré au-dessus de la tranchée,

- en présence d'un horizon peu perméable à faible profondeur provoquant un écoulement hypodermique dans une couche superficielle motteuse.

\subsubsection{La persistance des produits phytosanitaires}

Celle-ci est estimée classiquement par la demi-vie du produit (DT50) : ce terme désigne la durée nécessaire pour que $50 \%$ de la quantité initiale disparaisse, aussi bien du fait de sa mobilité que de sa dégradation. La DT50 varie également considérablement suivant les molécules : de quelques jours à quelques centaines de jours.

Le ruissellement, mouvement très rapide de l'eau, produit ainsi un transfert en général significativement plus important que l'infiltration vers les couches profondes, mouvement beaucoup plus lent (sauf conditions extrêmes). Les écoulements subsuperficiels se situent certainement entre les deux, mais restent mal connus. Des résultats obtenus sur réseau de drainage montrent des départs rapides quand une pluie survient peu de temps après l'application, en période de fonctionnement des drains [2,3].

D'une manière générale, quelle que soit la DT50 de la molécule, la part la plus importante du transfert apparaît à l'occasion des pluies qui surviennent dans les premières semaines qui suivent l'application, ceci, dans la mesure où ces pluies produisent un écoulement d'eau hors de la tranche de sol efficace pour retenir et dégrader les molécules. C'est pourquoi la contamination par les herbicides, généralement appliqués entre la fin de l'automne et le printemps, est plus importante que celle produite par les fongicides, d'utilisation globalement plus estivale.

Ainsi, la date d'application, en relation avec les conditions climatiques, joue un rôle essentiel, relativement indépendant de la DT50 théorique des molécules.

Les produits qui ne sont pas évacués rapidement par les écoulements qui suivent les premières pluies sont stockés dans la microporosité du sol ou du substrat. Leur persistance joue alors un rôle discriminant : les molécules à faible DT50 vont disparaître des écoulements ultérieurs, alors que les molécules à DT50 élevée sont libérées, en produisant un " bruit de fond " qui, pour être très inférieur aux premières concentrations, reste souvent néanmoins suffisant pour engendrer un niveau de contamination significatif.

La comparaison de deux herbicides couramment utilisés, l'atrazine et l'isoproturon, de mobilité voisine (Koc d'environ 100 ) mais de persistance différente (respectivement une soixantaine et une vingtaine de jours) [4] est significative : le premier se retrouve toute l'année, alors que la présence du deuxième reste généralement limitée aux mois qui suivent l'application. Pour la même raison probablement, l'atrazine est un contaminant très fréquent dans les nappes aussi bien que dans les eaux de surface, alors que l'isoproturon est souvent identifié dans ces dernières et beaucoup plus rarement dans les nappes [5].

\subsection{Principales questions à résoudre}

De nombreux travaux (anciens, récents ou en cours) traitent des divers aspects qui viennent d'être abordés, à la fois sous l'angle de la description et de la modélisation des processus :

- concernant le ruissellement et l'infiltration, le fonctionnement du drainage,... pour ce qui a trait aux mouvements de l'eau ;

- concernant la mobilité et la dégradation des produits phytosanitaires, leur transfert par ruissellement, infiltration et drainage.

Toutefois, du fait des interactions complexes entre les facteurs climatiques, pédologiques, agronomiques et autres qui interviennent dans ces processus, il reste encore beaucoup à faire pour que ces connaissances soient réellement opérationnelles ; en particulier, il convient de s'intéresser à la variabilité temporelle et spatiale des processus en jeu.

Un compartiment du système mérite une mention particulière en terme de besoin de recherche : l'horizon labouré. En effet, cet horizon, qui joue un rôle stratégique de par sa position, est soumis à une forte variabilité temporelle et n'est encore l'objet que de très peu d'investigations. Sans prétendre être exhaustif, on peut citer quelques pistes de recherche, qui concernent à la fois les aspects hydrodynamiques et qualitatifs du fonctionnement de cet horizon :

- l'étude de la perméabilité et de l'importance du développement des semelles de labours ;

- la mise au point, pour les besoins de la modélisation, de relations décrivant la circulation de l'eau dans ce type de sol non saturé, en remplacement des classiques relations entre conductivité, succion et humidité (les courbes “ K-psitheta "), adaptées aux matériaux microporeux ;

- la connaissance des caractéristiques de sa macroporosité (liée aux mottes, aux résidus de culture) en relation avec sa conductivité subhorizontale et sa capacité à fixer et à dégrader les produits ;

- la répartition des produits dans le profil et leur disponibilité pour le transfert au moment des pluies.

Les questions concernant l'horizon labouré ne sont en fait qu'un cas particulier de celles que pose la circulation de l'eau dans tous les horizons où une macroporosité importante est présente. Mais la complexité y est particulièrement grande du fait du rôle essentiel qu'y jouent l'intervention humaine et les variations temporelles qui lui sont associées, ainsi que l'activité biologique qui s'y déroule.

\section{III — LE VERSANT}

\subsection{Le cheminement de l'eau}

Avant de constituer le réseau hydrographique proprement dit, les écoulements suivent les talwegs au sein même des parcelles cultivées. Ce sont les sous-bassins d'amont qui alimentent ces talwegs que l'on nomme ici " versants " (figure 3) [6]. Quand l'hydromorphie des sols est importante, les écoulements naturels sont modifiés par l'existence d'un réseau de fossés d'assainissement agricole, auquel s'ajoute le réseau de fossés de protection de la voirie. Ces fossés collectent et redirigent les eaux de ruissellement et, pour partie, les écoulements hypodermiques (quand le fond des fossés est en dessous du niveau de ces écoulements). 


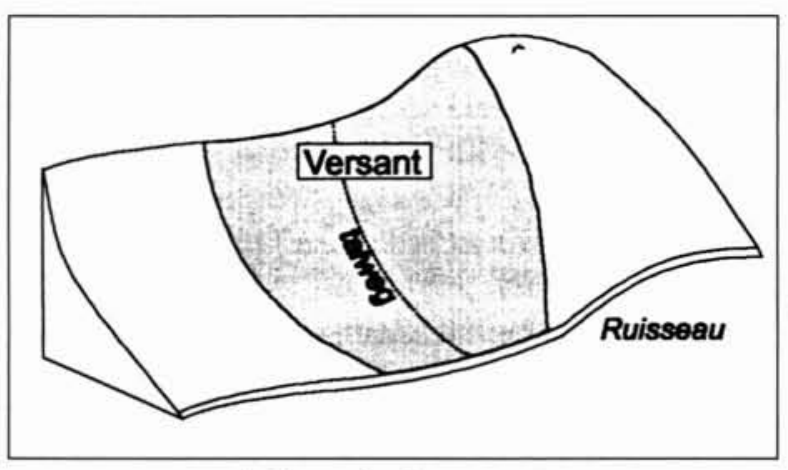

3. Exemple de versant.

D'autres éléments du paysage influent sur le devenir des écoulements provenant des parcelles :

- les talus, souvent associés à des fossés en zone bocagère, qui contribuent à rediriger et/ou à infiltrer le ruissellement. Les haies sans talus peuvent également jouer un rôle de réinfiltration,

- les " zones tampons", constituées par des prairies ou par des surfaces non cultivées : boisements, friches ou zones humides. Ces zones tampons jouent un rôle hydraulique en favorisant la réinfiltration du ruissellement des parcelles, ou au moins en ralentissant les écoulements (dans le cas des terrains imperméables ou humides).

\subsection{Le transfert des substances}

Les résidus végétaux et les sols très riches en humus ont un pouvoir de fixation des produits phytosanitaires supérieur à celui des sols cultivés [7]. La surface des zones tampons, généralement très organique, est donc susceptible de fixer les produits phytosanitaires entraînés par le ruissellement. Des expérimentations entreprises en France et aux USA ces dernières années ont montré que les zones tampons enherbées étaient effectivement aptes à réduire le transfert de ces molécules, et pas seulement celui des matières en suspension et des nutriments, ce qui est connu depuis plus longtemps [8]. Cette efficacité est expliquée pour partie par la réinfiltration, mais aussi par le pouvoir de fixation des surfaces en herbe. Elles contribuent donc à augmenter les possibilités de dégradation des produits avant qu'ils ne parviennent au réseau hydrographique.

En ce qui concerne les fossés, une première expérimentation réalisée récemment semble montrer également une certaine efficacité (cas d'un fossé riche en débris végétaux) [9]. Dans le cas des haies, aucune mesure n'a été effectuée, à notre connaissance.

Même si les zones tampons représentent une proportion réduite de la surface d'un bassin versant, elles peuvent jouer un rôle significatif sur le transfert des substances, à condition qu'elles occupent des emplacements stratégiques, c'està-dire au niveau des zones d'écoulement. Leur localisation en bordure de rivière, qui est le plus communément envisagée, protège celle-ci des transferts provenant des parcelles riveraines. Mais les écoulements qui y parviennent par l'intermédiaire de ruisseaux ou de fossés ne sont pas interceptés et le devenir des produits ainsi transférés ne dépend que de l'éventuel pouvoir d'épuration de ces collecteurs.

Cette question, essentielle sur le plan pratique, de la localisation des zones tampons en relation avec celle des fossés a été abordée récemment $[10,11]$, et devrait être développée.

\subsection{Principales questions à résoudre}

L'étude des relations hydrodynamiques entre le ruissellement et les écoulements hypodermiques d'un côté, et les éléments linéaires du paysage (fossés, haies, talus) de l'autre a été abordée anciennement mais reste également à développer [12].

La modélisation de ces relations, juste entreprise actuellement [13, 14], paraît indispensable pour, plus globalement, modéliser le transfert des produits phytosanitaires dans un bassin versant. Un modèle de ce type serait en effet bien utile pour simuler et comparer des scénarios d'aménagement à cette échelle.

Il reste à progresser en ce qui concerne l'effet des zones tampons enherbées sur le transfert des produits phytosanitaires, mais les résultats acquis ces dernières années permettent tout de même de préconiser leur mise en place [10]. Il existe par contre peu de références concernant les autres éléments du paysage cités ci-dessus : zones tampons boisées et humides, fossés, haies et talus.

\section{IV — LE BASSIN VERSANT}

Les principaux processus qui nous intéressent ici se produisent à l'échelle du versant. Le changement d'échelle conduit néanmoins à prendre en compte d'autres aspects, dont l'importance croît avec la dimension du bassin. Sans prétendre être exhaustif, nous citerons les points suivants :

- le rôle des échanges entre nappe et rivière : ces échanges existent déjà sur les petits bassins, mais prennent toute leur importance dans le cas des grands systèmes alluviaux,

- le rôle joué par les activités non agricoles et l'habitat urbain, rôle qui apparaît au moins sous deux formes différentes :

- l'apport au milieu aquatique de produits phytosanitaires d'origine non agricole (espaces verts, voirie, ...),

- le rejet dans le milieu aquatique de matières organiques très différentes des matières humiques qui dominent dans les bassins amont : rejets domestiques ou industriels, hydrocarbures, poussière provenant de l'usure des pneumatiques ou d'origine diverse,... Il est probable que ces substances aient des propriétés de fixation des produits phytosanitaires assez différentes de celles des substances humiques. Mais, à notre connaissance, il n'existe pas d'information à ce sujet.

Par ailleurs, pour proposer des solutions correctives sur un grand bassin (par exemple, une ressource en eau superficielle de quelques centaines de $\mathrm{km}^{2}$ ), il faut au préalable réaliser un diagnostic des causes de la contamination. Un document du CORPEN propose les bases d'un tel diagnostic [15]. Mais il n'aborde pas l'identification des voies superficielles de circulation de l'eau dans le bassin. Cet aspect n'est en effet pas nécessaire pour évaluer l'intérêt de méthodes de lutte en rapport avec les pratiques agricoles (raisonnement des traitements, pratiques de manipulation et d'application). Il est par contre indispensable pour évaluer le rôle joué par les zones tampons et les éléments linéaires (fossés, talus, haies) ou proposer leur mise en place ou la modification de l'occupation des sols. Outre le développement des connaissances abordé aux deux premières échelles, apparaît ici le besoin de méthodologies opérationnelles pour traiter cette question à faible coût sur une grande superficie. 


\section{$\mathrm{V} \square$ CONCLUSION}

Le devenir des produits utilisés par l'agriculture dépend bien évidemment de leurs propriétés. Mais, nous espérons l'avoir montré dans cet exposé, il est aussi très largement tributaire des caractéristiques du milieu où se produisent leur application et leur transfert.

Le cheminement de l'eau qui les transporte joue un rôle essentiel et reste encore trop mal connu, surtout dans les conditions propres aux sols cultivés : il existe en effet de multiples interactions entre les propriétés de ces substances, leur période d'application et les particularités des différents substrats où se produisent les écoulements.

Ainsi, de nombreux mécanismes qui interviennent dans ces processus de transfert restent à être quantifiés et décrits sous l'angle de leur variation spatio-temporelle pour asseoir les propositions de solutions correctives, que l'on s'appuie pour ce faire sur l'expertise ou la modélisation.

Pour conclure, nous insisterons sur les points pour lesquels des progrès rapides nous paraissent très souhaitables : ce sont, d'une part, les propriétés hydrodynamiques et physicochimiques de l'horizon cultivé et, d'autre part, le rôle joué par les zones tampons et les éléments linéaires du paysage.

\section{Bibliographie}

[1] WIDIATMAKA. 1994. Analyse structurale et fonctionnement hydrique d'un système pédologique limoneux acide sur granite et sur schiste du massif armoricain. INRA, laboratoire de sciences du sol. Thèse de l'université de Rennes. 260 p.

[2] BABUT M., SCHIAVON M., PORTAL J.M. 1996. La parcelle drainée : un outil pour l'évaluation du risque de pollution des eaux par les produits phytosanitaires. Actes du séminaire " Hydrosystèmes. Nancy, 2223 mai 1996. Pp. 127-140.

[3] REAL B. 1997. Site de la Jaillière ; rapport final ISMAP. Projet Eureka EU 479. 58p.
[4] DABENE E., MARIE F., SMITH C. 1995. Substances actives phytosanitaires : caractéristiques utiles pour l'évaluation du comportement de quelques substances actives dans l'environnement. Ministère de l'Agriculture - DERF.

[5] GREPPES (Région Centre). 1997. Bilan des actions 1991-96.

[6] FAN Y., BRAS R.L. 1998. Analytical solutions to hillslope subsurface storm flow and saturation overland flow. Water resources research. 34(4). pp. 921-927.

[7] BENOIT P., BARRIUSO E., VIDON P., REAL B. Isoproturon sorption and degradation in a soil from grassed buffer strip. J. Environ. Quality. A paraître.

[8] PATTY L. Limitation du transfert par ruissellement vers les eaux superficielles de deux herbicides. Méthodologie analytique et étude de l'efficacité de bandes enherbées. 1997. Cemagref- ITCF. Thèse de I'Université Joseph Fournier (Grenoble). 217 p.

[9] GARON-BOUCHER C. (1), CHARNAY L. (2). 1998. Rétention des produits phytosanitaires par les fossés : mises au point analytiques et expérimentations de terrain (1) ; rôle des sédiments (2). Cemagref. DEA de l'université Claude Bernard (Lyon). 43 p.(1) ; 53 p. (2).

[10] CORPEN. 1997. Produits phytosanitaires et dispositifs enherbés. Etat des connaissances et propositions de mise en cuvre. Ministères de l'Environnement et de l'Agriculture. $88 \mathrm{p}$.

[11] SOUILlER C., GRIL J.J., CHABALIER D., CLUZEAU D. Diagnostic de la contamination par les produits phytosanitaires et mise en cuvre de zones tampons : une méthode d'analyse des voies de circulation de l'eau sur un bassin versant. Communication au 28 ème congrès du Groupe Français des Pesticides, Alès, mai 1998. A parâtre.

[12] MEROT P., CARNET C., PIGNAULT, RUELLAN A. 1976. Rôle du bocage dans la circulation de l'eau : premiers résultats. Bulletin français de Pisciculture. 48 (261). Pp. 208-219.

[13] CARLUER N., GOUY V., GRIL J.J. 1996. Contamination des eaux de surface par les produits phytosanitaires et modélisation. Ingénieries EAT. $N^{\circ} 6$. pp. 19-30.

[14] CARLUER N. 1998. Vers une modélisation hydrologique adaptée à l'évaluation des pollutions diffuses : prise en compte du réseau anthropique. Application au bassin versant de Naizin (Morbihan), thèse Paris VI, 384 p.

[15] CORPEN, 1996. Qualité des eaux et produits phytosanitaires : propositions pour une démarche de diagnostic. Ministères de l'Environnement et de l'Agriculture. $120 \mathrm{p}$. 